Research Paper

\title{
The association of HLA/KIR genes with non-small cell lung cancer (adenocarcinoma) in a Han Chinese population
}

\author{
Yingfu Li2², Shuyuan Liu1, Chao Hong1, Qianli Ma³, Fang Tan², Chengxiu Liu1, Piotr Kuśnierczyk4, \\ Chuanyin $\mathrm{Li}^{1}$, Li Shi ${ }^{\bowtie}$ and Yufeng Yao ${ }^{\boxplus}$ \\ 1. Institute of Medical Biology, Chinese Academy of Medical Sciences \& Peking Union Medical College, Kunming 650118, China \\ 2. Department of Geriatrics, The No.1 Affiliated Hospital of Kunming Medical University, Kunming 650032, China \\ 3. Department of Thoracic Surgery, The No.3 Affiliated Hospital of Kunming Medical University, Kunming 650118, China \\ 4. Laboratory of Immunogenetics and Tissue Immunology, Ludwik Hirszfeld Institute of Immunology and Experimental Therapy, Polish Academy of \\ Sciences, Wrocław, Poland \\ *Yingfu Li and Shuyuan Liu contributed equally.
}

$\square$ Corresponding authors: Dr. Li Shi, Institute of Medical Biology, Chinese Academy of Medical Sciences \& Peking Union Medical College, NO. 935 Jiaoling Rd., Kunming 650118, Yunnan, China. Tel: +86 871 68335632; Fax: +86 871 68334483; Email address: shili.imb@gmail.com OR Dr. Yufeng Yao, Institute of Medical Biology, Chinese Academy of Medical Sciences \& Peking Union Medical College, NO. 935 Jiaoling Rd., Kunming 650118, Yunnan, China. Tel: +86 871 68335632; Fax: +86 871 68334483; Email address: leoyyf@gmail.com

() The author(s). This is an open access article distributed under the terms of the Creative Commons Attribution License (https://creativecommons.org/licenses/by/4.0/). See http://ivyspring.com/terms for full terms and conditions.

Received: 2019.01.28; Accepted: 2019.06.26; Published: 2019.08.20

\begin{abstract}
The host immune system plays a crucial role in the surveillance, recognition and elimination of tumor cells. Recent studies found that Human lymphocyte antigen class I (HLA I) genes, Killer cell immunoglobulin-like receptor $(K I R)$ genes and $H L A / K I R$ combinations play a role in the defense against tumor cells. To evaluated the associations between HLA I genes, KIR genes and HLA/KIR combinations and non-small cell lung cancer (NSCLC) in a Chinese Han population, a total of 229 patients with NSCLC (adenocarcinoma) and 217 healthy individuals were studied. Our results showed that the HLA-C*08:0I allele occurred at a significantly higher frequency in the NSCLCs compared with the controls $(P=0.034)$. The $H L A$ haplotype frequencies bearing $H L A-A$, $-B$, and $-C$ loci between the NSCLC and control groups were not different $(P>0.05)$. And there were no differences in the KIR gene, genotype and haplotype frequencies between the NSCLC and control groups $(P>0.05)$. Also, there were no differences between the HLA/KIR combinations in the KIR3D genes and HLA-A3/AII, HLA-Bw4 ligands and KIR2D genes and HLA-C1/C2 ligands between the NSCLC and control groups $(P>0.05)$. Our results indicate that the HLA-C*08:0I allele could be a risk factor for NSCLC (adenocarcinoma) in the Chinese Han population $(\mathrm{OR}=2.395 ; 95 \% \mathrm{Cl}: 1.359-4.221)$.
\end{abstract}

Key words: non-small cell lung cancer, HLA, KIR, HLA/KIR combination, Chinese Han population

\section{Introduction}

Lung cancer causes a large number of cancer-related deaths around the world, and its 5-year survival rate is approximately $15 \%$ [1]. Among lung cancer cases, non-small cell lung cancer (NSCLC) accounts for approximately $80 \%$ of lung cancer cases [2]. To date, more and more studies have proven that host genes play a key role in the susceptibility or development of NSCLC, especially host immune system genes.

The host immune system can recognize and eliminate tumor cells [3]; this is mediated by CD8 ${ }^{+}$ cytotoxic $\mathrm{T}$ cell adaptive immune responses. Human lymphocyte antigen class I molecules (HLA-I) play a key role in presenting tumor antigens for $\mathrm{CD} 8^{+}$ cytotoxic T cells recognition [4]. HLA-I molecules are encoded by HLA I genes; three classical transplantation HLA genes (HLA-A, HLA-B and $H L A-C)$ have been observed to have multiple roles in immune regulation [5].

Natural killer (NK) cells are crucial components of the innate immune system, which is involved in the immune response to different diseases including 
cancers [6]. Killer cell immunoglobulin-like receptors (KIRs) are present on NK cells, and located within the leukocyte receptor complex [7]. To date, 16 KIR gene loci have been identified, including two pseudogenes (KIR2DP1 and KIR3DP1), seven inhibitory KIR genes (KIR2DL1, KIR2DL2, KIR2DL3, KIR 2DL5, KIR3DL1, KIR3DL2 and KIR3DL3), six activating genes (KIR2D S1, KIR2DS2, KIR2DS3, KIR2DS4, KIR2DS5, and KIR3DS1), and KIR2DL4, whose product may have both inhibitory and activating capacities [8-10].

HLA-I molecules and KIR serve as ligands and receptors, respectively, and their interplay is important for transmitting activating or inhibitory signals to regulate the function of NK cells [11]. Their interactions are also a component of the innate immune system, which plays a role in the defense against tumor cells. Thus, the function of HLA I genes, $K I R$ genes and their combinations are valuable for investigating associations with susceptibility or worse clinical outcomes in different types of cancer, such as kidney cancer, breast cancer, colorectal cancer and lung cancer [12-18].

To investigate the role of genetic variations in HLA I genes, KIR genes and HLA/KIR combinations in the susceptibility or development of NSCLC, we evaluated the association of HLA I genes, KIR genes and HLA/KIR combinations with NSCLC (adenocarcinoma) in a Chinese Han population in this study.

\section{Material and Methods}

\section{Ethical approval and informed consent}

All procedures were in accordance with the ethical standards of the responsible committee on human experimentation and with the Helsinki Declaration of 1964, which was revised in 2013. All experimental protocols used in this study were approved by the Institutional Review Boards of the No. 3 Affiliated Hospitals of Kunming Medical University. All participants provided written informed consent.

\section{Subjects}

From December 2013 to January 2016, we selected 229 patients (134 males and 95 females) as the case group who were diagnosed with NSCLC and adenocarcinoma based on pathomorphological reports at the No. 3 Affiliated Hospitals of Kunming Medical University. The histological type and pathologic stage of the lung cancer was identified according to the World Health Organization (WHO 2004) classifications and the International System for Staging Lung Cancer [19]. The NSCLC patient inclusion criteria: 1 ) the patients were aged $\geq 18$ years with histologically and pathologically diagnosed NSCLC; 2) the patients were diagnosed with adenocarcinoma; 3 ) the patients had not received preoperative neoadjuvant therapies (including chemotherapy and radiotherapy). The criteria for the exclusion was 1) the patients with a prior history of primary cancer other than lung cancer; 2) the patients with small cell lung cancer; 3) the patient with malignant tumors except lung cancer; 4) the patients receiving radiotherapy or chemotherapy, and unclear pathological diagnosis. Clinical characteristics and data, such as sex, age, family history of cancer, and histological type of cancer, were obtained. The healthy controls included 217 subjects (143 males and 74 females) were recruited from a population undergoing routine examinations at the No.3 Affiliated Hospitals of Kunming Medical University. The controls underwent clinical examinations and did not have any cancer, or family history of NSCLC, or respiratory diseases and matched to the cases by age and gender. All participants (NSCLC patients and healthy controls) were unrelated Chinese Han individuals.

\section{HLA I genes (HLA-A, -B and -C) genotyping}

Blood samples were collected and genomic DNA was extracted from peripheral lymphocytes using the QIAamp Blood Kit (Qiagen, Hilden, Germany) according to the manufacturer's protocol. $H L A-A,-B$, and $-C$ genes were genotyped with the NGS sequencing-based HLA typing using the NGSgo $\mathrm{R}$ Illumina Miseq workflow (Illumina, Inc. San Diego, CA, USA) according to the manufacturer's instructions. Briefly, the NGSgo-AmpX kit was first used to perform HLA locus-specific ( $H L A-A,-B$, and $-C)$ amplification. Then, the large amplicons were randomly fragmented to $300-800 \mathrm{bp}$ prior to adapter ligation to ensure that the whole gene was evenly covered by numerous overlapping reads. The end-repair and dA-tailing were completed in the same step. After fragmentation, adapter ligation was performed, followed by a second clonal amplification and then sequencing. During sequencing, millions of reads were generated from multiple samples and multiple loci simultaneously. The NGS engine software package (Illumina) was used to determine the HLA genotypes for the individual samples and loci in an easy and user-friendly manner based on NGS data using the following three steps: (1) Alignment, (2) Haplotype phasing, and (3) Genotype determination.

\section{KIR gene genotyping}

In the current study, sixteen KIR genes (excluding 3DP1) [20] were genotyped using 
multiplex PCR sequence-specific priming (PCR-SSP) and identified by electrophoresis via agarose gels as described previously [21]. Two sets of primers were designed for each of the sixteen KIR genes and the presence of a gene was detected only when both were amplified.

\section{Statistical analysis}

The $H L A-A,-B$ and $-C$ allele frequencies were calculated with the Pypop and PyHLA software based on the genotyping results [22-24]. The $\chi^{2}$ test was used to determine differences in the allele frequencies between the NSCLC and healthy control groups. The odds ratios (OR) and associated 95\% confidence intervals (CIs) were also calculated for allele-specific risks. The Hardy-Weinberg equilibrium was assessed using the Guo and Thompson method [25]. The $H L A-A,-B$ and $-C$ haplotypes were constructed and calculated using the expectation-maximization algorithm based on the genotyping results.

For KIR loci, the frequency of each KIR gene was determined by direct counting. The genotypes and haplotypes were defined by referring to the Allele Frequencies website (http://www.allelefrequencies. net). The $\chi^{2}$ test was used to determine differences in gene, genotype and haplotype frequencies between the NSCLC and healthy control groups.

For $H L A / K I R$ combinations, the inhibitory KIR3DL1 recognizes $H L A-B B w 4$ and KIR3DL2 binds to HLA-A3 and HLA-A11 [26-28]. Based on the dimorphism in the epitope at position 80, all HLA-C alleles can be divided into the following two groups: (1) the $C 1$ group carrying asparagine and (2) the $C 2$ group carrying lysine. The $\mathrm{C} 1$ group consists of HLA-Cw1,-Cw3, -Cw7, -Cw8,-Cw12,-Cw13, and $-C w 14$. The C2 group consists of HLA-Cw2, -Cw4, $-C w 5,-C w 6,-C w 15$, and -Cw17. The HLA-C1 ligands bind to KIR2DL2, KIR2DL3 and KIR2DS2 receptors, whereas HLA-C2 ligands bind to KIR2DL1 and KIR2DS1 [26, 29, 30]. Thus, the HLA-A3/A11, Bw4, $\mathrm{C} 1$ and $\mathrm{C} 2$ groups were also used to analyze the HLA/KIR combinations. The differences in the HLA/KIR combination frequencies between the NSCLC and healthy control groups were determined by the $X^{2}$ test.

The false discovery rate (FDR) correction was used for the multiple comparisons [22, 31], and adjusted $P$ value less than 0.05 was considered statistically significant.

\section{Results}

\section{Subject characteristics}

Table 1 lists the characteristics of the enrolled subjects. There were no age or gender differences between the NSCLC and healthy control groups $(P>0.05)$.

\section{Association of HLA I genes and their haplotypes with NSCLC}

The allele frequencies for $H L A-A,-B$ and $-C$ in the NSCLC and healthy control groups are listed in Table 2. The genotype frequencies for $H L A-A,-B$ and $-C$ were in Hardy-Weinberg equilibrium for both the NSCLC and healthy control groups $(P>0.05)$. The frequencies of the $H L A-A^{*} 02: 06, B^{*} 13: 01, B * 40: 01$, and $C^{*}$ 08:01 alleles were different between the NSCLC and healthy control groups $(P<0.05)$. However, after FDR correction, only $H L A-C^{*} 08: 01$ occurred at a significantly higher frequency in the NSCLC group compared with the healthy control group $(P=0.034$, $\mathrm{OR}=2.395$; 95\% CI: 1.359-4.221). Additionally, there is no differentiation of the susceptibility between male and female $(P=0.482)$. The HLA haplotype analysis $(>1 \%)$ bearing HLA-A, $-\mathrm{B},-\mathrm{C}$ loci showed that the ten haplotypes $\left(A^{*} 02: 01-B^{*} 46: 01-C^{*} 01: 02, A^{*} 02: 03-B^{*} 15: 02-\right.$

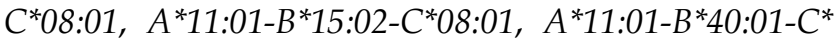
03:04, $A^{*} 11: 01-B^{*} 52: 01-C^{*} 12: 02, A^{*} 11: 01-B^{*} 54: 01-C^{*} 01$ : 02, $A^{*} 24: 02-B^{*} 15: 25-C^{*} 04: 03, A^{*} 24: 02-B^{*} 40: 01-C^{*} 07: 02$, $A^{*} 24: 02-B^{*} 51: 01-C^{*} 14: 02$ and $A^{*} 33: 03-B^{*} 44: 03-C^{*} 07: 01$ ) were different between the NSCLC and healthy control groups (Table 3), however, the significance of differences faded after FDR correction (Table 3). In the subtype analysis, there were differences in frequencies of $H L A-B^{*} 15: 11$ and haplotype $A^{*} 02: 01-B^{*}$ 15:11-C*03:03, $\quad A^{*} 11: 01-B^{*} 38: 02-C^{*} 07: 02, \quad A^{*} 2402-B^{*}$ 07:02-C*07:02 and $A^{*} 24: 02-B^{*} 46: 01-C^{*} 01: 02$ between pathologic stages I+II and III+IV (data not shown). However, after FDR correction, there were no differences in the HLA alleles and haplotypes between pathologic stages I+II and III+IV (data not shown).

Table 1. Clinical characteristics of the subjects enrolled in the present study

\begin{tabular}{llll}
\hline & NSCLC (n=229) & Control (n=217) & $\boldsymbol{P}$ value \\
\hline N & 229 & 217 & 446 \\
Ages ( years ) & $54.85 \pm 11.20$ & $54.42 \pm 7.46$ & 0.634 \\
Sex ( M/F ) & $134 / 95$ & $143 / 74$ & 0.108 \\
Adenocarcinoma (AD) & 229 & & \\
Clinical stage & & & \\
I & 38 & & \\
II & 39 & & \\
III & 66 & & \\
IV & 86 & \\
\hline
\end{tabular}

Notes: Data are mean \pm SD

\section{Association of $16 \mathrm{KIR}$ genes and their haplotypes with NSCLC}

All 16 KIR genes were detected in the NSCLC and healthy control groups. The four framework loci, KIR3DL3, KIR3DP1, KIR2DL4, and KIR3DL2, were 
observed in every individual in the NSCLC and healthy control groups. The KIR gene (KIR2DL2, KIR2DL5, KIR2DS2 and KIR2DS3) and genotype (5, 15,23 and 64) frequencies were different in the NSCLC and healthy control groups $(P<0.05)$. However, there were no significant differences in the KIR genes and genotypes between the NSCLC and healthy control groups after FDR correction (Tables 4 and 5). KIR haplotypes A and B showed no significant differences in the NSCLC and healthy control groups with frequencies of 0.459 vs. 0.525 for haplotype A and 0.541 vs. 0.475 for haplotype $B$, respectively $(P>0.05)$ (Supplementary Table 1$)$. In the subgroups analysis, only KIR genotype 9 showed differences between pathologic stages I+II and III+IV (data not shown). However, after FDR correction, there were no differences in the KIR gene, genotype and haplotype frequencies between pathologic stages I+II and III+IV (data not shown).

Table 2. HLA-A, HLA-B, and HLA-C allele frequencies in NSCLC group $(n=229)$ and healthy control group $(n=217)$

\begin{tabular}{|c|c|c|c|c|}
\hline Allele & NSCLC (freq) & Control (freq) & $P$ value & OR \\
\hline $\mathrm{A}^{*} 01: 01$ & 0.018 & 0.023 & 0.851 & 0.754 \\
\hline$A^{*} 02: 01$ & 0.118 & 0.085 & 0.721 & 1.434 \\
\hline$A^{*} 02: 03$ & 0.066 & 0.042 & 0.721 & 1.620 \\
\hline$A^{*} 02: 06$ & 0.018 & 0.053 & 0.111 & 0.318 \\
\hline$A * 02: 07$ & 0.107 & 0.097 & 0.851 & 1.118 \\
\hline$A^{*} 02: 10$ & 0.002 & 0.002 & 1.000 & 0.948 \\
\hline$A^{*} 03: 01$ & 0.011 & 0.028 & 0.721 & 0.388 \\
\hline$A^{*} 03: 02$ & 0.002 & 0.002 & 1.000 & 0.948 \\
\hline$A^{*} 11: 01$ & 0.273 & 0.277 & 1.000 & 0.982 \\
\hline$A^{*} 11: 02$ & 0.004 & 0.007 & 0.851 & 0.630 \\
\hline$A * 24: 02$ & 0.168 & 0.194 & 0.851 & 0.842 \\
\hline$A^{*} 24: 03$ & 0.009 & 0.005 & 0.851 & 1.903 \\
\hline$A * 24: 07$ & 0.013 & 0.007 & 0.851 & 1.907 \\
\hline$A * 26: 01$ & 0.031 & 0.018 & 0.851 & 1.679 \\
\hline$A * 29: 01$ & 0.009 & 0.002 & 0.851 & 3.815 \\
\hline$A * 30: 01$ & 0.031 & 0.025 & 0.851 & 1.213 \\
\hline$A * 31: 01$ & 0.031 & 0.018 & 0.851 & 1.679 \\
\hline$A * 32: 01$ & 0.007 & 0.012 & 0.851 & 0.566 \\
\hline$A * 33: 03$ & 0.076 & 0.088 & 0.851 & 0.862 \\
\hline$A^{* 68: 01}$ & 0.002 & 0.007 & 0.851 & 0.314 \\
\hline$A^{* 69: 01}$ & 0.004 & 0.005 & 1.000 & 0.947 \\
\hline $\mathrm{B}^{*} 07: 02$ & 0.015 & 0.012 & 1.000 & 1.332 \\
\hline$B^{*} 07: 05$ & 0.011 & 0.002 & 0.876 & 4.779 \\
\hline $\mathrm{B}^{*} 08: 01$ & 0.004 & 0.005 & 1.000 & 0.947 \\
\hline$B^{*} 13: 01$ & 0.046 & 0.090 & 0.373 & 0.487 \\
\hline$B^{*} 13: 02$ & 0.039 & 0.023 & 0.876 & 1.735 \\
\hline$B^{\star} 15: 01$ & 0.048 & 0.042 & 1.000 & 1.166 \\
\hline$B^{*} 15: 02$ & 0.070 & 0.042 & 0.726 & 1.736 \\
\hline $\mathrm{B}^{\star} 15: 07$ & 0.002 & 0.002 & 1.000 & 0.948 \\
\hline$B^{*} 15: 11$ & 0.013 & 0.018 & 1.000 & 0.707 \\
\hline$B^{*} 15: 12$ & 0.015 & 0.007 & 0.890 & 2.230 \\
\hline$B^{*} 15: 18$ & 0.004 & 0.002 & 1.000 & 1.899 \\
\hline $\mathrm{B}^{*} 15: 25$ & 0.018 & 0.018 & 1.000 & 0.947 \\
\hline$B^{*} 15: 27$ & 0.004 & 0.007 & 1.000 & 0.630 \\
\hline$B * 27: 04$ & 0.011 & 0.014 & 1.000 & 0.787 \\
\hline$B * 35: 01$ & 0.033 & 0.042 & 1.000 & 0.783 \\
\hline$B * 35: 05$ & 0.013 & 0.009 & 1.000 & 1.427 \\
\hline$B * 37: 01$ & 0.007 & 0.009 & 1.000 & 0.709 \\
\hline$B * 38: 02$ & 0.042 & 0.030 & 0.890 & 1.402 \\
\hline$B * 39: 01$ & 0.028 & 0.018 & 0.890 & 1.556 \\
\hline
\end{tabular}

\begin{tabular}{|c|c|c|c|c|}
\hline Allele & NSCLC (freq) & Control (freq) & $P$ value & OR \\
\hline$B^{*} 40: 01$ & 0.081 & 0.124 & 0.619 & 0.619 \\
\hline$B^{*} 40: 02$ & 0.022 & 0.012 & 0.876 & 1.915 \\
\hline$B^{\star} 40: 06$ & 0.028 & 0.016 & 0.876 & 1.782 \\
\hline$B^{*} 44: 02$ & 0.004 & 0.014 & 0.876 & 0.313 \\
\hline$B^{*} 44: 03$ & 0.015 & 0.028 & 0.876 & 0.546 \\
\hline$B^{*} 46: 01$ & 0.157 & 0.152 & 1.000 & 1.040 \\
\hline$B^{*} 48: 01$ & 0.007 & 0.021 & 0.726 & 0.311 \\
\hline$B * 50: 01$ & 0.004 & 0.002 & 1.000 & 1.899 \\
\hline$B * 51: 01$ & 0.055 & 0.032 & 0.876 & 1.732 \\
\hline$B * 51: 02$ & 0.011 & 0.007 & 1.000 & 1.586 \\
\hline$B * 52: 01$ & 0.033 & 0.035 & 1.000 & 0.946 \\
\hline$B * 54: 01$ & 0.046 & 0.044 & 1.000 & 1.050 \\
\hline$B * 55: 02$ & 0.024 & 0.028 & 1.000 & 0.865 \\
\hline$B \star 56: 01$ & 0.004 & 0.002 & 1.000 & 1.899 \\
\hline$B * 57: 01$ & 0.004 & 0.007 & 1.000 & 0.630 \\
\hline$B * 58: 01$ & 0.042 & 0.058 & 0.876 & 0.708 \\
\hline$C^{\star} 01: 02$ & 0.240 & 0.217 & 0.851 & 1.143 \\
\hline$C^{*} 03: 02$ & 0.044 & 0.062 & 0.851 & 0.688 \\
\hline$C^{*} 03: 03$ & 0.063 & 0.065 & 1.000 & 0.980 \\
\hline$C^{*} 03: 04$ & 0.085 & 0.124 & 0.499 & 0.655 \\
\hline$C^{\star} 04: 01$ & 0.055 & 0.060 & 1.000 & 0.906 \\
\hline$C * 04: 03$ & 0.020 & 0.032 & 0.851 & 0.601 \\
\hline$C^{\star} 05: 01$ & 0.004 & 0.005 & 1.000 & 0.947 \\
\hline$C * 06: 02$ & 0.055 & 0.046 & 1.000 & 1.195 \\
\hline$C^{\star} 07: 02$ & 0.173 & 0.148 & 0.851 & 1.205 \\
\hline$C^{\star} 07: 04$ & 0.009 & 0.002 & 0.851 & 3.815 \\
\hline$C^{\star} 08: 01$ & 0.094 & 0.042 & 0.034 & 2.395 \\
\hline$C^{\star} 12: 02$ & 0.026 & 0.039 & 0.851 & 0.660 \\
\hline$C^{\star} 12: 03$ & 0.022 & 0.023 & 1.000 & 0.946 \\
\hline$C^{*} 14: 02$ & 0.039 & 0.037 & 1.000 & 1.069 \\
\hline$C^{*} 14: 03$ & 0.004 & 0.002 & 1.000 & 1.899 \\
\hline$C^{*} 15: 02$ & 0.026 & 0.023 & 1.000 & 1.141 \\
\hline
\end{tabular}

Notes: The $P$ value is corrected by FDR correction

Table 3. Common HLA A-B-C haplotypes frequencies in NSCLC group $(n=229)$ and healthy control group $(n=217)$

\begin{tabular}{|c|c|c|c|}
\hline Haplotype & NSCLC (freq) & Control (freq) & $P$ value \\
\hline $\mathrm{A}^{*} 02: 01-\mathrm{B}^{*} 15: 11-\mathrm{C}^{*} 03: 03$ & 0.011 & 0.007 & $>0.05$ \\
\hline $\mathrm{A}^{*} 02: 01-\mathrm{B}^{*} 40: 01-\mathrm{C}^{*} 03: 04$ & 0.008 & 0.010 & $>0.05$ \\
\hline$A^{*} 02: 01-B^{*} 46: 01-C^{*} 01: 02$ & 0.000 & 0.012 & $>0.05$ \\
\hline $\mathrm{A}^{*} 02: 03-\mathrm{B}^{*} 15: 02-\mathrm{C}^{*} 08: 01$ & 0.015 & 0.000 & $>0.05$ \\
\hline $\mathrm{A}^{*} 02: 03-\mathrm{B}^{\star} 38: 02-\mathrm{C}^{*} 07: 02$ & 0.014 & 0.009 & $>0.05$ \\
\hline $\mathrm{A}^{*} 02: 07-\mathrm{B}^{*} 46: 01-\mathrm{C}^{*} 01: 02$ & 0.090 & 0.075 & $>0.05$ \\
\hline$A^{*} 11: 01-B^{\star} 13: 01-C^{*} 03: 04$ & 0.027 & 0.035 & $>0.05$ \\
\hline$A^{*} 11: 01-B^{*} 15: 01-C^{*} 04: 01$ & 0.012 & 0.009 & $>0.05$ \\
\hline$A^{*} 11: 01-B^{*} 15: 02-C^{*} 08: 01$ & 0.043 & 0.014 & $>0.05$ \\
\hline$A^{*} 11: 01-B^{*} 38: 02-C^{*} 07: 02$ & 0.013 & 0.007 & $>0.05$ \\
\hline$A^{*} 11: 01-B^{*} 40: 01-C^{*} 03: 04$ & 0.004 & 0.019 & $>0.05$ \\
\hline$A^{*} 11: 01-B^{*} 40: 01-C^{*} 07: 02$ & 0.033 & 0.023 & $>0.05$ \\
\hline$A^{*} 11: 01-B^{*} 46: 01-C^{*} 01: 02$ & 0.024 & 0.032 & $>0.05$ \\
\hline$A^{*} 11: 01-B^{*} 51: 01-C^{*} 14: 02$ & 0.018 & 0.014 & $>0.05$ \\
\hline$A^{*} 11: 01-B^{\star} 52: 01-C^{*} 12: 02$ & 0.000 & 0.012 & $>0.05$ \\
\hline$A^{*} 11: 01-B^{*} 54: 01-C^{*} 01: 02$ & 0.000 & 0.013 & $>0.05$ \\
\hline$A^{*} 11: 01-B^{*} 55: 02-C^{*} 01: 02$ & 0.013 & 0.008 & $>0.05$ \\
\hline$A^{*} 24: 02-B^{*} 13: 01-C^{*} 03: 04$ & 0.010 & 0.015 & $>0.05$ \\
\hline$A^{*} 24: 02-B^{*} 15: 25-C^{*} 04: 03$ & 0.003 & 0.016 & $>0.05$ \\
\hline$A^{*} 24: 02-B^{\star} 38: 02-C^{*} 07: 02$ & 0.013 & 0.002 & $>0.05$ \\
\hline$A^{*} 24: 02-B^{*} 39: 01-C^{*} 07: 02$ & 0.010 & 0.005 & $>0.05$ \\
\hline$A^{*} 24: 02-B^{*} 40: 01-C^{*} 03: 04$ & 0.012 & 0.002 & $>0.05$ \\
\hline$A^{*} 24: 02-B^{*} 40: 01-C^{*} 07: 02$ & 0.000 & 0.018 & $>0.05$ \\
\hline$A^{*} 24: 02-B^{*} 46: 01-C^{*} 01: 02$ & 0.030 & 0.015 & $>0.05$ \\
\hline$A^{*} 24: 02-B^{*} 51: 01-C^{*} 14: 02$ & 0.010 & 0.000 & $>0.05$ \\
\hline$A^{*} 24: 02-B^{\star} 54: 01-C^{*} 01: 02$ & 0.018 & 0.017 & $>0.05$ \\
\hline$A^{*} 30: 01-B^{*} 13: 02-C^{*} 06: 02$ & 0.024 & 0.021 & $>0.05$ \\
\hline 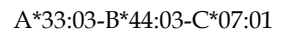 & 0.000 & 0.012 & $>0.05$ \\
\hline $\mathrm{A}^{*} 33: 03-\mathrm{B}^{*} 58: 01-\mathrm{C}^{*} 03: 02$ & 0.037 & 0.044 & $>0.05$ \\
\hline
\end{tabular}

Notes: The $P$ value is corrected by FDR correction 
Table 4. KIR genes frequencies in NSCLC group $(n=229)$ and healthy control group $(n=217)$

\begin{tabular}{llll}
\hline KIR locus & NSCLC (freq) & Control (freq) & $\boldsymbol{P}$ value \\
\hline 3DL1 & 0.934 & 0.945 & $>0.05$ \\
2DL1 & 0.991 & 1.000 & $>0.05$ \\
2DL3 & 0.991 & 0.982 & $>0.05$ \\
2DS4 & 0.939 & 0.945 & $>0.05$ \\
2DL2 & 0.297 & 0.180 & 0.064 \\
2DL5 & 0.441 & 0.313 & 0.075 \\
3DS1 & 0.393 & 0.336 & $>0.05$ \\
2DS1 & 0.393 & 0.332 & $>0.05$ \\
2DS2 & 0.258 & 0.180 & $>0.05$ \\
2DS3 & 0.223 & 0.143 & $>0.05$ \\
2DS5 & 0.262 & 0.286 & $>0.05$ \\
2DL4 & 1.000 & 1.000 & $>0.05$ \\
3DL2 & 1.000 & 1.000 & $>0.05$ \\
3DL3 & 1.000 & 1.000 & $>0.05$ \\
2DP1 & 0.991 & 1.000 & $>0.05$ \\
3DP1 & 1.000 & 1.000 & $>0.05$ \\
\hline
\end{tabular}

Notes: The $P$ value is corrected by FDR correction

Table 5. KIR genotypes frequencies in NSCLC group $(n=229)$ and healthy control group $(n=217)$

\begin{tabular}{|c|c|c|c|}
\hline Genotype & NSCLC (freq) & Control (freq) & $P$ value \\
\hline 1 & 0.454 & 0.512 & $>0.05$ \\
\hline 2 & 0.122 & 0.088 & $>0.05$ \\
\hline 3 & 0.026 & 0.023 & $>0.05$ \\
\hline 4 & 0.070 & 0.037 & $>0.05$ \\
\hline 5 & 0.039 & 0.005 & $>0.05$ \\
\hline 6 & 0.017 & 0.009 & $>0.05$ \\
\hline 7 & 0.017 & 0.009 & $>0.05$ \\
\hline 8 & 0.066 & 0.074 & $>0.05$ \\
\hline 9 & 0.013 & 0.014 & $>0.05$ \\
\hline 11 & 0.004 & 0.000 & $>0.05$ \\
\hline 13 & 0.017 & 0.014 & $>0.05$ \\
\hline 14 & 0.000 & 0.014 & $>0.05$ \\
\hline 15 & 0.000 & 0.028 & $>0.05$ \\
\hline 17 & 0.004 & 0.005 & $>0.05$ \\
\hline 18 & 0.004 & 0.000 & $>0.05$ \\
\hline 23 & 0.004 & 0.037 & $>0.05$ \\
\hline 24 & 0.004 & 0.005 & $>0.05$ \\
\hline 28 & 0.000 & 0.005 & $>0.05$ \\
\hline 31 & 0.004 & 0.000 & $>0.05$ \\
\hline 33 & 0.004 & 0.000 & $>0.05$ \\
\hline 44 & 0.000 & 0.014 & $>0.05$ \\
\hline 55 & 0.000 & 0.005 & $>0.05$ \\
\hline 64 & 0.031 & 0.000 & $>0.05$ \\
\hline 68 & 0.013 & 0.023 & $>0.05$ \\
\hline 69 & 0.013 & 0.009 & $>0.05$ \\
\hline 70 & 0.004 & 0.005 & $>0.05$ \\
\hline 73 & 0.000 & 0.005 & $>0.05$ \\
\hline 74 & 0.009 & 0.000 & $>0.05$ \\
\hline 75 & 0.004 & 0.014 & $>0.05$ \\
\hline 117 & 0.004 & 0.000 & $>0.05$ \\
\hline 156 & 0.004 & 0.000 & $>0.05$ \\
\hline 180 & 0.000 & 0.014 & $>0.05$ \\
\hline 200 & 0.004 & 0.000 & $>0.05$ \\
\hline 202 & 0.004 & 0.014 & $>0.05$ \\
\hline 232 & 0.000 & 0.005 & $>0.05$ \\
\hline 280 & 0.004 & 0.000 & $>0.05$ \\
\hline 294 & 0.004 & 0.000 & $>0.05$ \\
\hline 308 & 0.004 & 0.000 & $>0.05$ \\
\hline 326 & 0.000 & 0.005 & $>0.05$ \\
\hline 328 & 0.000 & 0.005 & $>0.05$ \\
\hline 370 & 0.009 & 0.000 & $>0.05$ \\
\hline 372 & 0.009 & 0.000 & $>0.05$ \\
\hline 381 & 0.004 & 0.000 & $>0.05$ \\
\hline 400 & 0.000 & 0.009 & $>0.05$ \\
\hline
\end{tabular}

Notes: The $P$ value is corrected by FDR correction
Associations of HLA/KIR combinations with NSCLC

The distribution of the two KIR3D genes and their HLA-A3/A11 or HLA-B Bw4 ligands is given in Supplementary Table 2. There were no significant differences $(P>0.05)$ in their combination frequencies between the NSCLC and healthy control groups. The distribution of the KIR2D genes and their HLA-C1/C2 ligands is given in Supplementary Table 2. The results for the KIR2D genes and their HLA-C1/C2 ligands showed that there were no significant differences in the frequencies between the NSCLC and healthy control groups $(P>0.05)$.

Recent evidence has demonstrated associations between HLA I genes, KIR genes and HLA/KIR combinations and autoimmune disease, infectious disease and cancers [12-18, 32-37]. In this study, we examined the role of genetic variations in HLA I genes, KIR genes and HLA/KIR combinations in the development of NSCLC in a Chinese Han population.

NSCLC is one of the most immunogenic tumors, and its tumor antigens can potentially be recognized by CTLs and $\mathrm{CD} 8^{+}$cytotoxic $\mathrm{T}$ cells, which mediate antitumor responses [38]. In 2010, Yang et al [39] investigated $H L A-A^{*} 02: 01, A^{*} 26: 01, B^{*} 15: 18$, and $B * 38: 02$ were associated with lung cancer in a Chinese Han population from North China. In the current study, we found that the frequencies of $H L A-A^{*} 02: 06$, $B^{*}$ 13:01 and $B^{*} 40: 01$ were lower in the NSCLC group than in the healthy control group (0.018 vs. 0.053 , 0.046 vs. 0.090 and 0.081 vs. 0.124 , respectively) (Table $2)$, though there were no differences between the two groups after FDR correction. Nevertheless, our results showed that $H L A-C^{*} 08: 01$ occurred at a significantly higher frequency in the NSCLC group compared with the healthy control group and showed a significant difference between the two groups after FDR correction (OR=2.395; 95\% CI: 1.359-4.221). One of the reasons for this discrepancy in the results between the Yang study and our study could be related to differences in sample sizes and pathomorphological type. In the current study, we enrolled 229 NSCLC patients and 217 healthy individuals, while only 100 lung cancer patients were included in Yang's study (81 NSCLC and 19 small cell lung cancer patients). Additionally, our NSCLC group only included adenocarcinoma patients, while Yang's study included lung cancer patients who were not further classified by pathomorphological type. Another reason might be the genetic differences between the North Han and Yunnan Han population. In 2015, Zhou et al. investigated the distribution of HLA allele and haplotype frequencies in the Chinese population, and found there were regional differences in the HLA diversities in China [40]. Moreover, our previous 
study also showed Han Chinese populations were divided into northern and southern clusters based on $H L A-A,-B$, and -DRB1 allele frequencies using phylogenetic tree and multidimensional scaling analyses [41]. The Han population from Yunnan is situated between the northern and southern clusters and showed different genetic characteristics from the Northern Han population [41]. The third reason could be because there was no correction for multiple comparisons in Yang's study, but we used FDR for multiple comparisons in the current study. In 2009, Nagata et al [42] reported that $H L A-A^{*} 02$ and $H L A-A^{*} 24$ could be prognostic factors for Japanese patients with NSCLC. Then, Bulut et al [43] investigated that $H L A-A^{*} 02$ was an independent risk factor for lymph node and distant metastases in patients with NSCLC in Turkish populations. Additionally, they also found that $H L A-A^{*} 26$ appeared to be a protective allele against metastases. However, we did not observe these differences in the HLA I gene allele and haplotype frequencies between pathologic stages I+II and III+IV after multiple comparison correction.

To escape $\mathrm{CD}^{+}$cytotoxic $\mathrm{T}$ cell recognition, tumor cells must eliminate HLA I molecules by downregulating their expression at the cell surface [44]. However, if HLA expression is completely lacking, NK cells will attack and kill tumor cells. Thus, variations in KIR genes have been thought as a major risk for developing cancer [45, 46]. In 2010, Al Omar et al [17] reported that there were no significant differences in the proportion of patients with different KIR genes, genotypes and haplotypes among patients with solid tumors (NSCLC, small-cell lung cancer, colon cancer and kidney cancer). Then, $\mathrm{Yu}$ et al reported the KIR gene frequency showed no differences between NSCLC and healthy controls in a Chinese Han population [14]. Also Wiśniewski et al [13] did not find such differences in Polish Caucasians. Our results were similar to the Al Omar, $\mathrm{Yu}$ and Wiśniewski results in that we also found no differences in the KIR gene, genotype and haplotype frequencies. We also found that there were no differences in the KIR gene, genotype and haplotype frequencies between pathologic stages I+II and III+IV after multiple comparison correction.

\section{Discussion}

Several studies have reported that certain HLA/KIR combinations are associated with susceptibility and worse clinical outcomes and response to treatment in several types of cancer, including breast cancer, colorectal cancer and lung cancer [12-18]. In 2010, Al Omar et al performed an association study on $H L A / K I R$ combinations with
NSCLC; they found that NSCLC patients showed a significant increase in the frequency of KIR2DL1-C2 and decrease in the frequency of KIR2DL3-C1 in homozygotes [17]. However, in the current study, our results did not show any differences in the HLA/KIR frequencies between the NSCLC and healthy controls in the Chinese Han population, which was similar to a report by Wiśniewski for Polish Caucasians [13]. These authors observed only that HLA-C heterozygotic genotypes encoding both $C 1$ and $C 2$ ligand epitopes for KIR2DL2/3 and KIR2DL1 inhibitory receptors, respectively, were less frequent in patients than in controls, whereas opposite was true for $\mathrm{C} 1 \mathrm{C} 1$ and $\mathrm{C} 2 \mathrm{C} 2$ homozygotes. However, this association of KIR ligands was independent from their respective receptors or any other KIR genes [13]. In the present report, even when subgroup analysis was performed, there were no differences in the HLA/KIR combinations between pathologic stages I+II and III+IV.

Nevertheless, Wiśniewski et al found that NSCLC patients positive for the KIR2DL2 and KIR2DS2 genes and homozygous for C1 were 6 times more likely to respond to treatment than those with other genotypes $(P=0.034)$. In accordance with these results, patients with the KIR2DL2+/KIR2DS2+-C1C1 genotype survived longer than others $(P=0.009)$ [13]. However, $\mathrm{Yu}$ et al did not observe any relationship between $H L A / K I R$ combinations and response to treatment in NSCLC in a Chinese Han population [14]. Here, we did not examine the response to NSCLC treatment, which is one of limitations of the current study; thus, we could not compare our results with others. However, we did not observe any differences in the HLA/KIR combinations between pathologic stages I+II and III+IV.

We used the association studies to investigate the relationship between HLA/KIR and NSCLC in the current study. One of the disadvantages in the association study is the high probability of false positive. Several factors influence the rate of false positive, such as population heterogeneity and stratification, and multiple testing. In order to avoid population heterogeneity and stratification, in this current study, we recruited the Han Chinese from the same region only, in addition, we restricted the pathomorphological to adenocarcinoma and made a series of inclusion and exclusion criteria for the case and control group which matched each other in gender and age. For the multiple testing, we used FDR correction for multiple comparisons and considered adjusted $P$ value less than 0.05 statistically. FDR is a method of conceptualizing the rate of type I error in null hypothesis testing when conducting multiple comparisons. FDR-controlling procedures 
provide less stringent control of Type I errors compared to family-wise error rate (FWER) controlling procedures and have greater power at the cost of increased numbers of Type I errors [31].

Another limitation of the current study was smoking history, which is a key risk factor for NSCLC and was not included in the data derived from the healthy control individuals in the current study. Although about $85-90 \%$ of lung cancer cases are smokers, the frequency of this malignancy among non-smokers seems to rise [47]. Several genetic polymorphisms have been recently found to be associated with lung cancer among non-smoking Chinese women [48, 49], including those not exposed to cooking oil fume [50, 51]. For Brasilian lung cancer patients (mostly with adenocarcinomas), different rates of EFGR and KRAS mutations were found in smokers and non-smokers [52]. The lack of smoking status for our healthy control individuals and patients makes it difficult to perform analyses that include such exposure variables and to perform a gene-smoking interaction analysis. Therefore, we are planning to recruit sufficient numbers of non-smoker NSCLC patients and comparable number of non-smoker controls to examine genetic risk factors independent of smoking.

Our interesting finding that $H L A-C^{*} 08: 01$ allele is associated with NSCLC in Yunnan Han Chinese is novel, as such a detailed study has not been so far performed in this or other populations (Pubmed search, 3 January, 2019). In addition, this association could hardly be detected, for example, in Caucasians, as $H L A-C^{*} 08: 01$ is very rare there, in contrast to $H L A-C^{*} 08: 02$, more frequent in Europeans but extremely rare in Chinese (www.allelefrequencies. net). In 2016, Eric Tran et al reported that they identified a polyclonal $\mathrm{CD} 8^{+} \mathrm{T}$-cell response against mutant KRAS G12D in tumor-infiltrating lymphocytes, and observed regression of lung metastases after the infusion of $H L A-C^{*} 08: 02-$ restricted tumor-infiltrating lymphocytes that were composed of four different T-cell clonotypes that specifically targeted KRAS G12D [53]. The molecules encoded by these two alleles differ to some extent in their peptide-binding motifs and therefore might also differ in their roles in the immune surveillance of cancer [54].

\section{Conclusion}

In the current study, we performed an association study between HLA I genes, KIR genes and HLA/KIR combinations and NSCLC in a Chinese Han population and found that $H L A-C^{*} 08: 01$ is a risk factor for NSCLC (adenocarcinoma). In the future, larger scale studies are needed to better clarify and examine the association between HLA I genes, KIR genes and HLA/KIR combinations and NSCLC susceptibility, resistance and disease progression.

\section{Supplementary Material}

Supplementary tables.

http://www.jcancer.org/v10p4731s1.pdf

\section{Acknowledgments}

We express our gratitude to all our patients and healthy control volunteers for kindly donating their blood and their agreement to participate in this study. This study was supported by National Natural Science Foundation of China (81573206 and 31270030), Yunnan Applied Basic Research Projects (2016FA034), Special Funds for high-level health talents of Yunnan Province (D-201669 and L-201615), the Association Foundation Program of the Yunnan Provincial Science and Technology Department and Kunming Medical University (No.2017FE468-193), and the Polish National Science Centre (No. 2014/15/B/ NZ5/03517). We also express the gratitude to Beijing Bofurui Gene Diagnostic Co., LTD for the technical support and service for HLA genotyping.

\section{Competing Interests}

The authors have declared that no competing interest exists.

\section{References}

1. Parkin DM, Bray F, Ferlay J, Pisani P. Global cancer statistics, 2002. CA Cancer J Clin. 2005; 55: 74-108.

2. Johnson JL, Pillai S, Chellappan SP. Genetic and biochemical alterations in non-small cell lung cancer. Biochem Res Int. 2012; 2012: 940405.

3. Dunn GP, Old LJ, Schreiber RD. The immunobiology of cancer immunosurveillance and immunoediting. Immunity. 2004; $21: 137-48$.

4. Leone P, Shin EC, Perosa F, Vacca A, Dammacco F, Racanelli V. MHC class I antigen processing and presenting machinery: organization, function, and defects in tumor cells. J Natl Cancer Inst. 2013; 105: 1172-87.

5. Shiina T, Hosomichi K, Inoko H, Kulski JK. The HLA genomic loci map: expression, interaction, diversity and disease. Journal of Human Genetics. 2009; 54: 15-39.

6. Caligiuri MA. Human natural killer cells. Blood. 2008; 112: 461-9.

7. Vilches C, Parham P. KIR: diverse, rapidly evolving receptors of innate and adaptive immunity. Annu Rev Immunol. 2002; 20: 217-51.

8. Wilson MJ, Torkar M, Haude A, Milne S, Jones T, Sheer D, et al. Plasticity in the organization and sequences of human KIR/ILT gene families. Proc Natl Acad Sci U S A. 2000; 97: 4778-83.

9. Biassoni R, Cantoni C, Falco M, Verdiani S, Bottino C, Vitale M, et al. The human leukocyte antigen (HLA)-C-specific "activatory" or "inhibitory" natural killer cell receptors display highly homologous extracellular domains but differ in their transmembrane and intracytoplasmic portions. J Exp Med. 1996; 183: 645-50.

10. Biassoni R, Cantoni C, Pende D, Sivori S, Parolini S, Vitale M, et al. Human natural killer cell receptors and co-receptors. Immunol Rev. 2001; 181: 203-14.

11. Lanier LL. NK cell recognition. Annual review of immunology. 2005; 23: 225-74.

12. De Re V, Caggiari L, De Zorzi M, Talamini R, Racanelli V, M DA, et al. Genetic diversity of the KIR/HLA system and outcome of patients with metastatic colorectal cancer treated with chemotherapy. PLoS One. 2014; 9: e84940.

13. Wisniewski A, Jankowska R, Passowicz-Muszynska E, Wisniewska E, Majorczyk E, Nowak I, et al. KIR2DL2/S2 and HLA-C C1C1 genotype is associated with better response to treatment and prolonged survival of patients with non-small cell lung cancer in a Polish Caucasian population. Hum Immunol. 2012; 73: 927-31.

14. Yu H, Liu F, Sansas B, Kang B, Preville X, Wu X, et al. Typing of killer-cell immunoglobulin-like receptors and their cognate human leukocyte antigen 
class I ligands predicts survival of Chinese Han patients with metastatic non-small-cell lung cancer. Mol Clin Oncol. 2017; 6: 279-85.

15. Kim HJ, Choi HB, Jang JP, Baek IC, Choi EJ, Park M, et al. HLA-Cw polypmorphism and killer cell immunoglobulin-like receptor (KIR) gene analysis in Korean colorectal cancer patients. Int J Surg. 2014; 12: 815-20.

16. Jobim MR, Jobim M, Salim PH, Portela P, Jobim LF, Leistner-Segal S, et al. Analysis of KIR gene frequencies and HLA class I genotypes in breast cancer and control group. Hum Immunol. 2013; 74: 1130-3.

17. Al Omar S, Middleton D, Marshall E, Porter D, Xinarianos G, Raji O, et al. Associations between genes for killer immunoglobulin-like receptors and their ligands in patients with solid tumors. Hum Immunol. 2010; 71: 976-81.

18. Middleton D, Vilchez JR, Cabrera T, Meenagh A, Williams F, Halfpenny I, et al. Analysis of KIR gene frequencies in HLA class I characterised bladder, colorectal and laryngeal tumours. Tissue Antigens. 2007; 69: 220-6.

19. Groome PA, Bolejack V, Crowley JJ, Kennedy C, Krasnik M, Sobin LH, et al. The IASLC Lung Cancer Staging Project: validation of the proposals for revision of the $\mathrm{T}, \mathrm{N}$, and $\mathrm{M}$ descriptors and consequent stage groupings in the forthcoming (seventh) edition of the TNM classification of malignant tumours. J Thorac Oncol. 2007; 2: 694-705.

20. Hsu KC, Liu XR, Selvakumar A, Mickelson E, O'Reilly RJ, Dupont B. Killer Ig-like receptor haplotype analysis by gene content: evidence for genomic diversity with a minimum of six basic framework haplotypes, each with multiple subsets. J Immunol. 2002; 169: 5118-29.

21. Kulkarni S, Martin MP, Carrington M. KIR genotyping by multiplex PCR-SSP. Methods Mol Biol. 2010; 612: 365-75.

22. Fan Y, Song YQ. PyHLA: tests for the association between HLA alleles and diseases. BMC Bioinformatics. 2017; 18: 90.

23. Lancaster AK, Single RM, Solberg OD, Nelson MP, Thomson G. PyPop update--a software pipeline for large-scale multilocus population genomics. Tissue Antigens. 2007; 69 Suppl 1: 192-7.

24. Lancaster A, Nelson MP, Meyer D, Thomson G, Single RM. PyPop: a software framework for population genomics: analyzing large-scale multi-locus genotype data. Pacific Symposium on Biocomputing Pacific Symposium on Biocomputing. 2003; 8: 514-25.

25. Guo SW, Thompson EA. Performing the exact test of Hardy-Weinberg proportion for multiple alleles. Biometrics. 1992; 48: 361-72.

26. Kulkarni S, Martin MP, Carrington M. The Yin and Yang of HLA and KIR in human disease. Semin Immunol. 2008; 20: 343-52

27. Gumperz JE, Litwin V, Phillips JH, Lanier LL, Parham P. The Bw4 public epitope of HLA-B molecules confers reactivity with natural killer cell clones that express NKB1, a putative HLA receptor. J Exp Med. 1995; 181: 1133-44.

28. Cella M, Longo A, Ferrara GB, Strominger JL, Colonna M. NK3-specific natural killer cells are selectively inhibited by Bw4-positive HLA alleles with isoleucine 80. J Exp Med. 1994; 180: 1235-42.

29. Parham P. MHC class I molecules and KIRs in human history, health and survival. Nat Rev Immunol. 2005; 5: 201-14.

30. Kim S, Sunwoo JB, Yang L, Choi T, Song YJ, French AR, et al. HLA alleles determine differences in human natural killer cell responsiveness and potency. Proc Natl Acad Sci U S A. 2008; 105: 3053-8.

31. Shaffer JP. Multiple Hypothesis Testing. Annual Review of Psychology. 1995; 46: 561-84

32. Martin MP, Qi Y, Gao X, Yamada E, Martin JN, Pereyra F, et al. Innate partnership of HLA-B and KIR3DL1 subtypes against HIV-1. Nat Genet. 2007; 39: 733-40.

33. Martin MP, Gao X, Lee JH, Nelson GW, Detels R, Goedert JJ, et al. Epistatic interaction between KIR3DS1 and HLA-B delays the progression to AIDS. Nat Genet. 2002; 31: 429-34.

34. Khakoo SI, Thio CL, Martin MP, Brooks CR, Gao X, Astemborski J, et al. HLA and NK cell inhibitory receptor genes in resolving hepatitis $C$ virus infection. Science. 2004; 305: 872-4

35. Karlsen TH, Boberg KM, Olsson M, Sun JY, Senitzer D, Bergquist A, et al. Particular genetic variants of ligands for natural killer cell receptors may contribute to the HLA associated risk of primary sclerosing cholangitis. J Hepatol. 2007; 46: 899-906.

36. van der Slik AR, Koeleman BP, Verduijn W, Bruining GJ, Roep BO, Giphart MJ. KIR in type 1 diabetes: disparate distribution of activating and inhibitory natural killer cell receptors in patients versus HLA-matched control subjects. Diabetes. 2003; 52: 2639-42

37. Yen JH, Moore BE, Nakajima T, Scholl D, Schaid DJ, Weyand CM, et al. Major histocompatibility complex class I-recognizing receptors are disease risk genes in rheumatoid arthritis. J Exp Med. 2001; 193: 1159-67.

38. Vogelstein B, Papadopoulos N, Velculescu VE, Zhou S, Diaz LA, Jr., Kinzler KW. Cancer genome landscapes. Science. 2013; 339: 1546-58.

39. Yang L, Wang LJ, Shi GL, Ni L, Song CX, Zhang ZX, et al. Analysis of HLA-A, HLA-B and HLA-DRB1 alleles in Chinese patients with lung cancer. Genet Mol Res. 2010; 9: 750-5.

40. Zhou XY, Zhu FM, Li JP, Mao W, Zhang DM, Liu ML, et al. High-Resolution Analyses of Human Leukocyte Antigens Allele and Haplotype Frequencies Based on 169,995 Volunteers from the China Bone Marrow Donor Registry Program. PLoS One. 2015; 10: e0139485.

41. Yao Y, Shi L, Shi L, Matsushita M, Yu L, Lin K, et al. Distribution of HLA-A,-B, - CW, and -DRB1 alleles and haplotypes in an isolated Han population in Southwest China. Tissue Antigens. 2009; 73: 561-8.
42. Nagata Y, Hanagiri T, Mizukami M, Kuroda K, Shigematsu Y, Baba T, et al. Clinical significance of HLA class I alleles on postoperative prognosis of lung cancer patients in Japan. Lung Cancer. 2009; 65: 91-7.

43. Bulut I, Meral M, Kaynar H, Pirim I, Bilici M, Gorguner M. Analysis of HLA class I and II alleles regarding to lymph node and distant metastasis in patients with non-small cell lung cancer. Lung Cancer. 2009; 66: 231-6.

44. Moller P, Hammerling GJ. The role of surface HLA-A,B,C molecules in tumour immunity. Cancer Surv. 1992; 13: 101-27.

45. Augusto DG. The Impact of KIR Polymorphism on the Risk of Developing Cancer: Not as Strong as Imagined? Front Genet. 2016; 7: 121.

46. Bashirova AA, Martin MP, McVicar DW, Carrington M. The killer immunoglobulin-like receptor gene cluster: tuning the genome for defense. Annu Rev Genomics Hum Genet. 2006; 7: 277-300.

47. Pelosof L, Ahn C, Gao A, Horn L, Madrigales A, Cox J, et al. Proportion of Never-Smoker Non-Small Cell Lung Cancer Patients at Three Diverse Institutions. J Natl Cancer Inst. 2017; 109.

48. Yin $\mathrm{Z}$, Zhou B, He O, Li M, Guan P, Li X, et al. Association between polymorphisms in DNA repair genes and survival of non-smoking female patients with lung adenocarcinoma. BMC Cancer. 2009; 9: 439.

49. Yin $\mathrm{Z}$, Cui $\mathrm{Z}$, Guan $\mathrm{P}, \mathrm{Li} \mathrm{X}, \mathrm{Wu} \mathrm{W}$, Ren $\mathrm{Y}$, et al. Interaction between Polymorphisms in Pre-MiRNA Genes and Cooking Oil Fume Exposure on the Risk of Lung Cancer in Chinese Non-Smoking Female Population. PLoS One. 2015; 10: e0128572.

50. Yin Z, Su M, Li X, Li M, Ma R, He Q, et al. ERCC2, ERCC1 polymorphisms and haplotypes, cooking oil fume and lung adenocarcinoma risk in Chinese non-smoking females. J Exp Clin Cancer Res. 2009; 28: 153.

51. Shen L, Yin Z, Wu W, Ren Y, Li X, Zhou B. Single nucleotide polymorphism in ATM gene, cooking oil fumes and lung adenocarcinoma susceptibility in Chinese female non-smokers: a case-control study. PLoS One. 2014; 9: e96911.

52. Bacchi CE, Ciol H, Queiroga EM, Benine LC, Silva LH, Ojopi EB. Epidermal growth factor receptor and KRAS mutations in Brazilian lung cancer patients. Clinics (Sao Paulo). 2012; 67: 419-24.

53. Tran E, Robbins PF, Lu YC, Prickett TD, Gartner JJ, Jia L, et al. T-Cell Transfer Therapy Targeting Mutant KRAS in Cancer. N Engl J Med. 2016; 375: 2255-62.

54. Rasmussen M, Harndahl M, Stryhn A, Boucherma R, Nielsen LL, Lemonnier FA, et al. Uncovering the peptide-binding specificities of HLA-C: a general strategy to determine the specificity of any MHC class I molecule. J Immunol. 2014; 193: 4790-802. 